\title{
Pulmonary Intravascular Hemangiosarcoma in a Cat
}

\author{
Tetsushi YAMAGAMI ${ }^{1)}$, Kouji NOMURA ${ }^{1)}$, Michiro FUJITA ${ }^{2)}$, Kiyokazu OZAKI ${ }^{3)}$, Hiromitsu ORIMA ${ }^{2)}$ and \\ Isao NARAMA ${ }^{3)}$
}

${ }^{1)}$ Marupi-lifetech Co. Ltd., 103 Fushio-cho, Ikeda, Osaka 563-0011, ${ }^{2}$ Department of Veterinary Radiology, Nippon Veterinary and Life Science University, 1-7-1 Kyonancho, Musashino, Tokyo 180-8602 and ${ }^{33}$ Department of Pathology, Faculty of Pharmaceutical Sciences, Setsunan University, 45-1 Nagaotouge-cho, Hirakata, Osaka 573-0101, Japan

(Received 29 October 2005/Accepted 1 March 2006)

ABSTRACT. A 9-year-old, castrated male Japanese domestic cat presented with a complaint of exertional dyspnea. Based on the radiographic findings, presumptive diagnosis of aspiration pneumonia or primary diffuse pulmonary neoplasia in the right middle lobe was made. Histologically, the pulmonary lesion was characterized by diffuse thickening of alveolar wall with the proliferation of apparently atypical irregular-shaped cells. Immunohistochemical staining using anti-human factor VIII-related antigen antiserum showed positive reaction in the cytoplasm of the atypical cells. According to the findings, the lesion was diagnosed as pulmonary intravascular hemangiosarcoma.

KEY WORDS: feline, hemangiosarcoma, lung.

Hemangiosarcoma is a malignant tumor derived from vascular endothelium. The malignant type of the tumor in cats have been less frequently reported compared to those in dogs, and the lesions were usually seen in the skin of the head, distal limbs and paws [3]. The lesion is often solitary, but sometime multicentric. The authors describe the clinical and pathological findings of pulmonary hemangiosarcoma observed in a cat with lesions of unusual intravascular diffuse pattern.

A 9-year-old, castrated male, Japanese domestic cat presented with exertional dyspnea. Neither traumatic lesion nor history of traffic accident had been recorded. Laboratory results including complete blood cell counts and biochemical analyses revealed a mild reactive anemia (RBC $5.12 \times 10^{6} / \mu l$, Ht 33\%) and a slight elevation of hepatic and renal enzymes (ALT $96 \mathrm{U} / l$, AST $53 \mathrm{U} / l$, BUN $98 \mathrm{mg} / \mathrm{d} l$, Cre $2.3 \mathrm{mg} / \mathrm{d} l)$.

Ventro-dorsal thoracic radiograph exhibited consolidation of the right middle lobe of the lung, and mixed image pattern of alveolar and unstructured-interstitional ones at the right cranial and caudal lobes (Fig. 1). Based on the radiographic findings, presumptive diagnosis of aspiration pneumonia or primary diffuse pulmonary neoplasia in the right middle lobe was made. In addition, foci of pneumonia at the cranial and caudal lobe were also suspected. Although thoracotomy was performed to remove the pulmonary lesion, the cat was died soon after the operation.

Surgically resected right posterior lobe revealed no obvious nodular lesions by gross examination. Tissue samples were fixed in $10 \%$ buffered formalin, routinely processed for histopathological examination. Paraffin sections were stained with hematoxylin and eosin (HE). Immunohistochemical staining was also performed using anti-human factor VIII-related antigen and anti-cytokeratin (AE1/AE3) sera (DAKO corp., Carpinteria, CA, U.S.A.) by avidinbiotin-peroxidase complex method.
Histologically, the lesion in the resected lode was characterized by diffuse thickening of alveolar wall (Fig. 2). The alveolar wall consisted of apparently atypical irregularshaped cells (Fig. 3). These cells had basophilic cytoplasm and a large irregular round-shaped nucleus. Large single or multiple nucleoli were prominent in the nucleus. Abnormal mitotic figures were frequently observed (Fig. 4). In some regions, atypical cells proliferated in small to medium sized vascular lumens (Fig. 5). Immunohistochemical staining using anti-human factor VIII-related antigen antiserum showed positive reaction in the cytoplasm of the atypical cells (Fig. 6). However, the cells were negative for cytokeratin (AE1/AE3)(Fig.7).

According to the findings, the tumor was diagnosed as intravascular hemangiosarcoma. The lesion was thought to be primary, because there were no clinical and radiographical evidences of neoplastic lesions in other organs.

Canine hemangiosarcoma is not a rare tumor, and

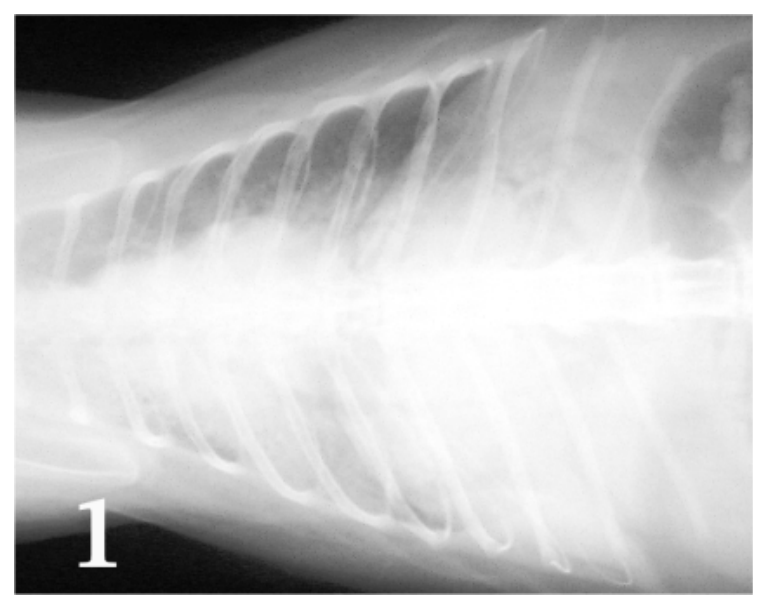

Fig. 1. Ventro-dorsal thoracic radiograph of the case. 


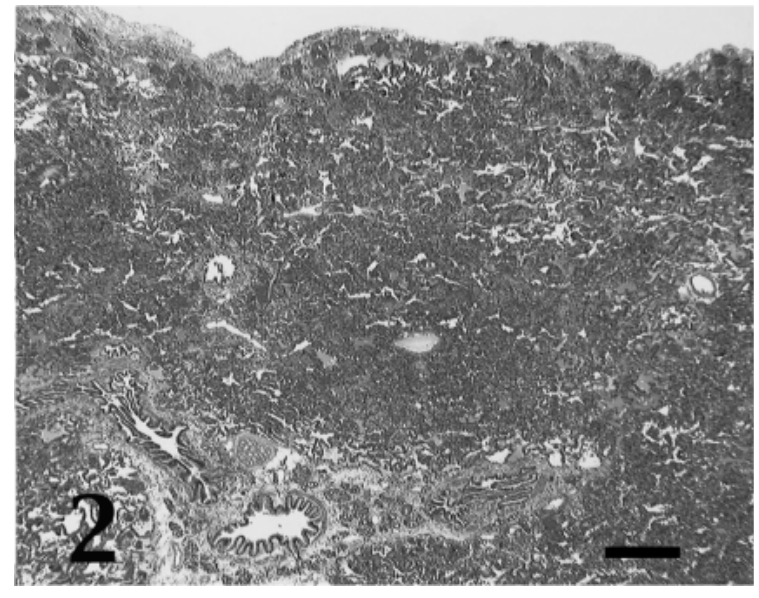

Fig. 2. Low magnification of right posterior lode. Note diffuse thickening of alveolar wall. HE, Bar=100 $\mu \mathrm{m}$.

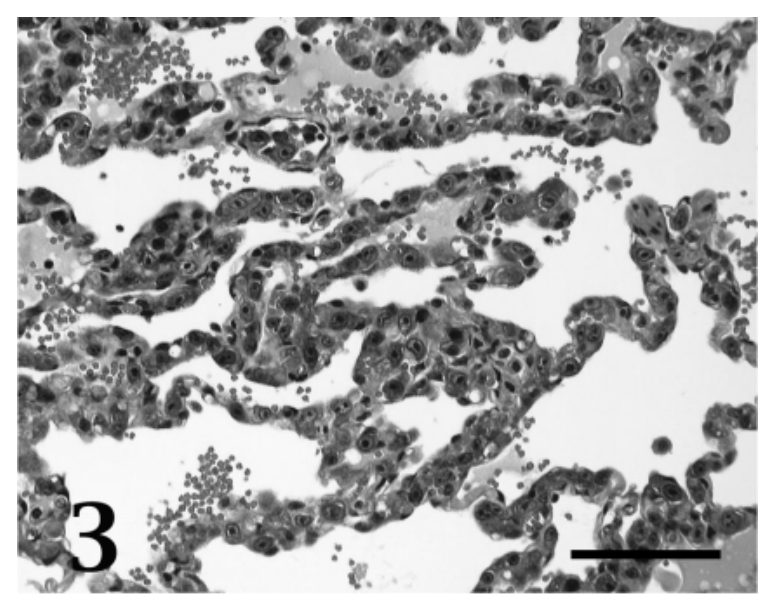

Fig. 3. Thickened alveolar wall consisted of apparently atypical irregular-shaped cells. HE, Bar $=100 \mu \mathrm{m}$.

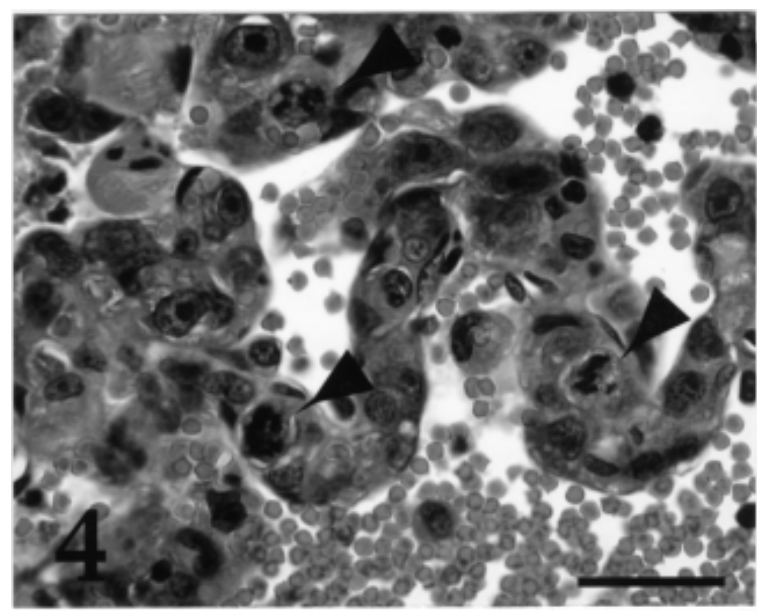

Fig. 4. Higher magnification of the atypical cells. Many abnormal mitotic figures (arrow heads) were observed in the alveolar septa. HE, Bar $=50 \mu \mathrm{m}$.

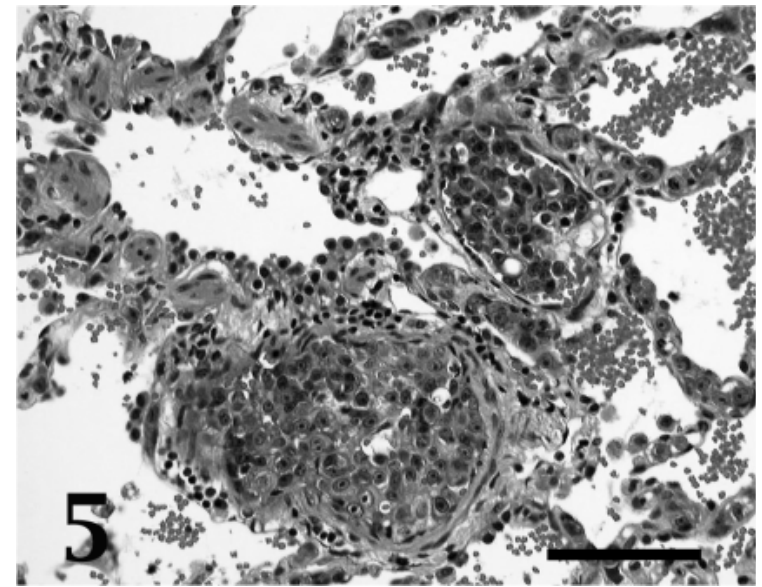

Fig. 5. Intravascular proliferation of the atypical cells in small- to medium-sized vascular lumens. $\mathrm{HE}, \mathrm{Bar}=100 \mu \mathrm{m}$.

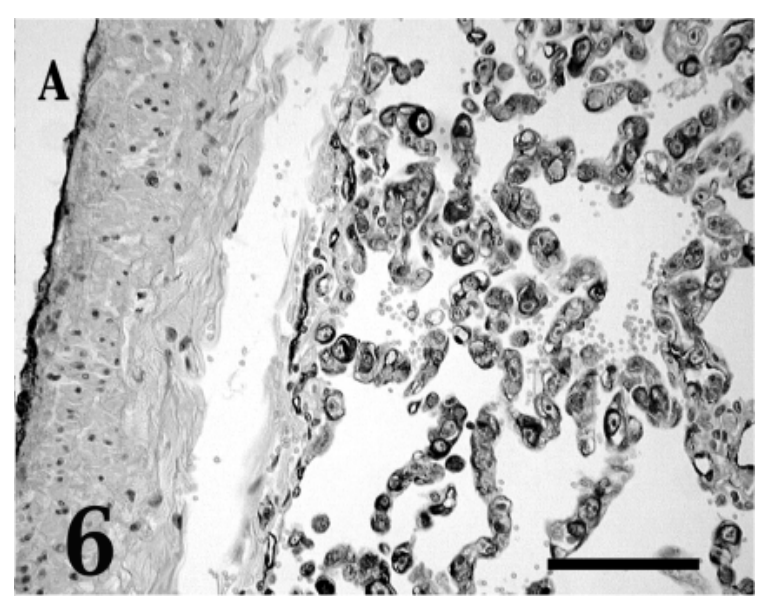

Fig. 6. The atypical cells are positive for human factor VIIIrelated antigen. Immunohistochemistry. Avidin-biotin-peroxidase complex method. A: arterial lumen. Bar $=100 \mu \mathrm{m}$.

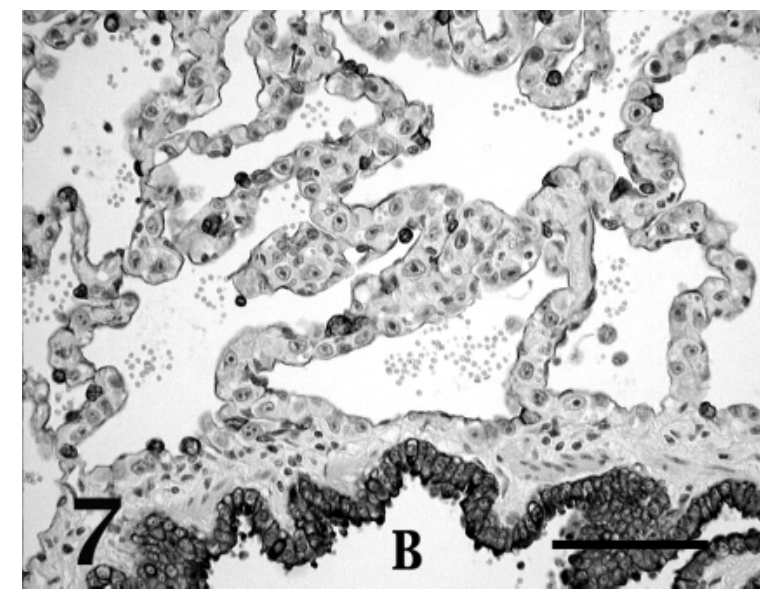

Fig. 7. Atypical cells were negative for cytokeratin (AE1/AE3). Immunohistochemistry. Avidin-biotin-peroxidase complex method. B: bronchial lumen. Bar=100 $\mu \mathrm{m}$. 
observed especially in the German shepherd and Golden retriever. The tumor arises from the spleen, liver, lung and right auricle. Although the tumor is less frequently in the cat, some have been reported in the head (especially eyelid), distal limbs and paws [4]. Primary mesenchymal tumors including osteosarcoma, chondrosarcoma, fibrosarcoma and undifferentiated sarcoma in the lung have been described in some animal species, but primary pulmonary hemangiosarcoma is extremely rare in domestic animals $[1,3]$. Histopathological features of primary pulmonary hemangiosarcomas in human [5] were quite different from those in the present feline case.

Although feline intravascular proliferative disorders are extremely rare, some cases of feline systemic reactive angioendotheliomatosis (FSRA) have been described [2]. FSRA are characterized by multisystemic intraluminal endothelial and pericyte proliferation without cellular atypia, and the heart is the most commonly and severely affected. No similar syndrome has been identified in humans. The present case was also characterized by intraluminal endothelial proliferation, but the differencies are atypia of proliferating endothelial cells and localized lesions in the lung.

Metastatic pulmonary tumor lesions usually form multiple nodules even in cases of intrapulmonary metastases.
The neoplastic cells, however, formed no massive lesions in the present case. In addition, neoplastic cells did not destroy alveolar structure. These may suggest that the tumor was originated in the lung and the tumor cells proliferated completely in the vascular lumen, even in small capillaries of the alveolar wall as well. The histopathological growth pattern of the tumor cells supports the radiographical diffuse pattern of pulmonary lesions. When a diffuse pattern of pulmonary lesion is observed by radiography, diffuse neoplasms including hemangiosarcoma should be considered in addition to aspiration pneumonia.

\section{REFERENCES}

1. Dungworth, D.L. 1993. pp. 688-696. In: Pathology of Domestic Animals, Fourth ed., vol.2 (Jubb, K.V.F., Kennedy, P.C. and Palmer, N. eds.), Academic Press, Inc., San Diego, CA.

2. Fuji, R.N., Patton, K.M., Steinbach, T.J., Schulman, F.Y., Bradley, G.A., Brown, T.T., Wilson, E.A. and Summers, B.A. 2005. Vet. Pathol. 42: 608-617.

3. Goldschmidt, M.H. and Hendrick, M.J. 2002. pp. 99-101. In: Tumors in Domestic Animals, 3rd ed. (Meuten, D.J. ed.), Iowa State Press, Iowa.

4. Moran, C.A. and Suster, S. 2001. pp. 184-189, In: Diagnostic Histopathology of Tumors, vol.1 (Fletcher, C.D.M., ed.), Churchill livingstone, London, UK.

5. Yousem, S.A. 1986. Arch. Pathol. Lab. Med. 110: 112-115. 